\title{
Secondary Stress Relaxation Mechanism in Ion-Containing Polymers I. A New Analytical Method
}

\author{
Hiroshi MatsuURA \\ Department of Research and Development, Canadian Technical Tape Ltd., \\ 455 Cote Vertu, St-Laurent, Quebec, Canada H4N 1 E8
}

(Received June 3, 1986)

\begin{abstract}
With a view to isolating the secondary component from the complexed stress relaxation, a method originally proposed by Tobolsky and used for the study of the chemical stress relaxation in covalently bonded networks was applied to ion-containing polymers to which timetemperature superposition had been previously found inapplicable. A few examples were chosen from the two different groups of ionomers to demonstrate the applicability of this method. Although the secondary component in one of styrene-sodium methacrylate copolymers (PSNaMA) with $7.7 \mathrm{~mol} \%$ of ions cannot be separated by this method at low temperatures, the secondary mechanism can be isolated at high temperatures. In other ionomers with more ions, the secondary mechanism is isolated by this method for the entire temperature ranges. The secondary relaxation is due to the yielding of the ionic structure, and these ionomers studied here approximately follow Maxwellian decay of stress. The activation energy obtained for the secondary mechanism of each ionomer, which ranges from 26 to $31 \mathrm{kcal} \mathrm{mol}^{-1}$, may correspond to the energy required to break the ionic interactions in the ionic clusters.
\end{abstract}

KEY WORDS Stress Relaxation / Relaxation Mechanism / Secondary

Relaxation / Ionomers / Styrene Ionomers / Acrylate Ionomers / Ionic

Clusters / Activation Energy /

One of the main problems complicating the interpretation of viscoelastic behaviors in the ionomers with high $\mathrm{mol} \%$ of ions is the fact that time-temperature superposition has, by and large, been found inapplicable. No simple master curve can be obtained from the stress relaxation of such ionomers. The long term viscoelastic behaviors of ionomers must result from two basically different relaxation mechanisms. Firstly, there can be ordinary physical relaxation process, such as the flow of polymer chains, the movement of entanglements, $\cdots$ etc. Secondly, there can be another process which must be attributed to the yielding of the ionic structures, besides true chemical process, such as the scission of covalent bonds. In general, the physical mechanism can be observed at low temperature range or short time region. On the other hand, the secondary mechanism, if it is due to the yielding of the ionic structures, can be observed at high temperature range or long time region. Of course, at certain temperature ranges and in certain time regions, there must be two independent relaxation mechanisms operative simultaneously. The main objective of this study is to illustrate a method whereby these two different relaxation mechanisms can be separated or, at least, the secondary one can be distinguished better.

There are basically two different approaches to separate or isolate the secondary process from the physical one. Firstly, both of them can be separated under certain conditions where details of the chemical process are known. For example, Tobolsky and others ${ }^{1}$ recognized and isolated the chemical com- 
ponent by performing the stress relaxation experiments at temperatures sufficiently high that the physical process could be neglected. Tobolsky and co-workers ${ }^{1}$ had indeed investigated various mechanisms of degradation, cross-linking, and bond-interchange in polymers with using this procedure. Bueche ${ }^{2}$ was able to attribute the chemical processes in the ordinary impure polydimethylsiloxanes to rearrangements caused by the presence of impurities by performing the stress relaxation experiments on purified samples. For many years, Murakami and co-workers ${ }^{3,4}$ have carried out a number of relaxation experiments on various cross-linked and non-crosslinked rubbers under controlled conditions, such as nitrogen atmosphere, to subtract the physical mechanism and isolate the chemical one. The procedures described above could be termed as "chemical approach."

Secondly, many workers were able to distinguish the chemical or secondary process differently by applying the time-temperature superposition principle to the stress relaxation data obtained from certain temperature range or time scale where the chemical or secondary process can be neglected. The WLF constants and other physical parameters were first determined and then used to extract the chemical or secondary components. For example, Ferry and co-workers ${ }^{5}$ utilized this procedure when they studied the stress relaxation of methacrylate polymers with relatively long side chains in which two relaxation mechanisms, one with the main chains and the other with the side chains, are affected differently by a change in temperature. More recently, Curro and Salazar ${ }^{6}$ distinguished the physical and the chemical relaxation mechanisms, using stress relaxation experiments as a function of temperature. The WLF constants and other physical parameters were first determined from experiments where the chemical process can be neglected over the time scale of the experiment. The physical contribution to the stress relaxation was then calculated for higher temperature experiments in order to extract the chemical kinetic information. The methods described above could be termed as "physical approach."

Previously, the "physical approach" has been almost exclusively used for the study of the stress relaxation in the ionomers. For example, Eisenberg and Navratil ${ }^{7-11}$ investigated the viscoelastic behavior of various salts of styrene-methacrylic acid copolymers by applying time-temperature superposition to the stress relaxation data obtained from them. They found time-temperature superposition is obeyed in the styrene ionomers below a critical ion concentration $\left(6 \mathrm{~mol}^{\circ} \%\right)$. The shift factors are of the WLF form although they are bigger than those of pure polystyrene. Above that ion concentration, however, time-temperature superposition becomes inapplicable. The results reported were in agreement with their hypothesis that the ionic sites of the polymer chains and the metal ions form ion pairs and these ion pairs are thought to associate further into ion multiplets. They ${ }^{9,11}$ also proposed that the ions exist in a form of simple multiplets below the critical ion concentration and in a form of more extensive clusters above that ion concentration. Eisenberg and $\mathrm{Navratil}^{9}$ claimed that the failure of time-temperature superposition indicates the existence of two independent relaxation mechanisms above such critical ion concentration. They also claimed that contributions from each mechanism can be separated and two "pseudo master curves" can be obtained ${ }^{11}$ by applying timetemperature superposition to the stress relaxation obtained from above the critical ion concentration. They defined, based on analysis of these two "master curves" and their respective shift factors, the viscoelastic responses in the short time segments as the physical nature for the entire temperature range and those in the long time segments as the secondary one.

More recently, Eisenberg, Matsuura, and 
Tsutui ${ }^{12}$ investigated the stress relaxation mechanism of various copolymers of ethyl. acrylate-acrylic acid salt. They found that, in this ionomer group, time-temperature superposition breaks down around $12 \mathrm{~mol} \%$ of ions. They reported that time-temperature superposition is generally applicable below $12 \mathrm{~mol} \%$ of ions.

The method $^{9-12}$ used by Eisenberg and coworkers is actually the same method that Ferry and co-workers ${ }^{5}$ utilized. There are, however, significant differences between the clustered ionomers and the methacrylate polymers. ${ }^{5}$ First of all, the clustered ionomers are, in contrast to the latter, not single phase polymer. ${ }^{13-15}$ In modulus-temperature curves of the clustered ionomers, ${ }^{9,12}$ the shape of the curves is not indicative of a truly amorphous polymer. The absence of a sharp transition region is very noticeable. Thus, the validity of applying the time-temperature superposition principle is questioned. With the ionic structures and large quantity of intermolecular associations present, relaxation processes are probably not confined to one simple mechanism. Thus, it was decided to analyze or isolate the mechanism which is due to the ionic clusters first by applying the "chemical approach," to the relaxation data obtained mainly at high temperatures. The contribution from pure physical process could be minimal at high temperatures and the secondary mechanism could be isolated by the "chemical approach." If the mechanism is really due to the yielding process of the ionic structure, similar to network cleavage or bond interchange type ${ }^{1}$ the stress relaxation must follow Maxwellian decay of stress. ${ }^{1}$

In this paper, the author shall report on the application of the "chemical approach" for the analysis of the secondary relaxation in the ionomers. Previously, the same data were analyzed by the "physical approach" and reported in the separate publication. ${ }^{9,12}$ The activation energies obtained by the "chemical approach" and the nature of the relaxation will also be discussed in this paper.

\section{EXPERIMENTAL}

The data previously obtained from the two different ionomer groups were utilized in this study: three PS-NaMA samples and one ethyl acrylate-sodium acrylate copolymer (PEA$\mathrm{NaA})$ sample. Original stress relaxation experiments were performed on PS-NaMA samples by Eisenberg and Navratil, and the results have been presented in the previous publications. $^{7-10}$ The preparation of the PSNaMA samples and the method of the stress relaxation experiments are described elsewhere. ${ }^{8}$ The original experiments with PEA$\mathrm{NaA}$ sample were carried out by the author and Eisenberg. ${ }^{12,13}$ The results analyzed by the "physical approach" were reported previously. ${ }^{12}$ Methods of both sample preparation and the stress relaxation experiments performed on PEA-NaA sample are described elsewhere. ${ }^{12,13}$

\section{RESULTS AND DISCUSSION}

Stress relaxation data obtained from one of the ionomers studied here, PS-NaMA $(7.7 \mathrm{~mol} \%$ of NaMA), are shown in Figure 1 to demonstrate how time-temperature superposition fails above the critical ion concentration $(6 \mathrm{~mol} \%$ in this case). It is apparent that the individual curves cannot be superimposed horizontally to form a true master curve no matter what time region is taken. Also shown in Figure 1 is a "pseudomaster curve" which was constructed by rather forcible superposition of the short time regions $(2-100 \mathrm{~s})$ of the individual curves. The failure of time-temperature superposition in the treatment of the stress relaxation data in the other ionomers studied here is basically the same as the one shown in Figure 1.

The lack of superposability must be attributed to the presence of multiple relaxation mechanisms. Furthermore, the existence of 


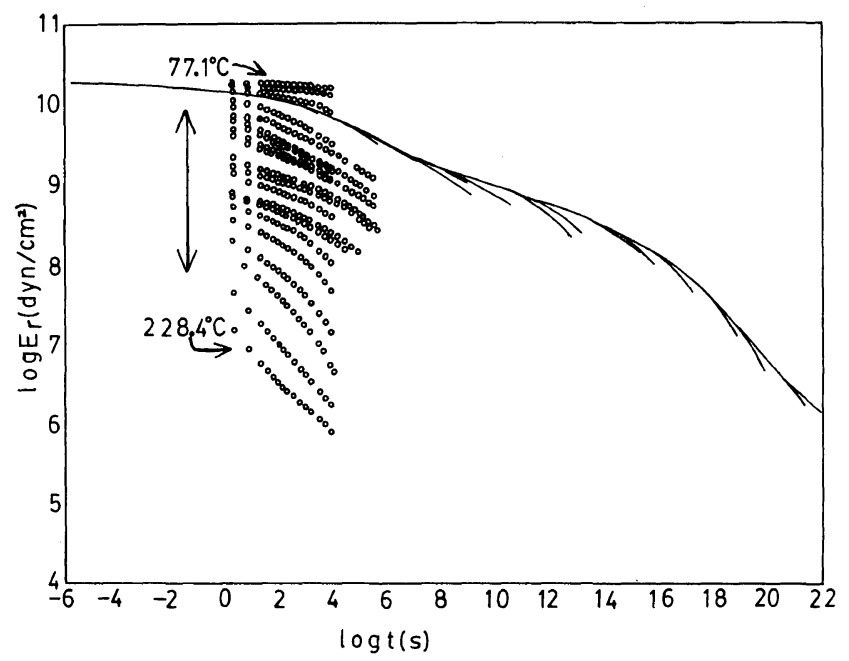

Figure 1. Original stress relaxation curves and "master curve" for PS-NaMA $(7.7 \mathrm{~mol} \%$ of NaMA). Time segment of $2-100 \mathrm{~s}$ was taken to construct the "master curve," reduced with respect to $T_{\mathrm{g}}$.

the secondary mechanism can be seen for almost entire temperature range, except around the sample's $T_{g}$ range, as evidenced by the downward deviation at long time regions for almost any given constant temperature on the "pseudo master curve" in Figure 1. The pure physical mechanism, even around the $T_{g}$, seems to exist in a relatively short period of time.

Contrary to the results obtained from both PS-NaMA ${ }^{8-9}$ and PEA-NaA ${ }^{12}$ by applying time-temperature superposition, what Ferry and co-workers ${ }^{5}$ found in their investigation of $N$-butyl methacrylate polymer is the fact that time-temperature superposition is generally well applicable at temperatures between 45 and $135^{\circ} \mathrm{C}$, except between 55 and $80^{\circ} \mathrm{C}$ at which some minor deviations are observed. Its superposability, above 80 and below $55^{\circ} \mathrm{C}$, is more than good enough to produce a pair of reasonable WLF constants and other physical parameters. Without reasonable physical parameters, it is difficult to extract the secondary mechanism by subtracting the physical component from the entire stress relaxation. When Salazar and Curro $^{6}$ identified the chemical process, they utilized some temperature range above the $T_{g}$ over which the chemical effects can be neglected for the time scale of their experiments (about $100 \mathrm{~h}$ ). It is obvious that long enough physical process over fairly wide temperature range is necessary to obtain reliable parameters.

At any given temperature, as can be seen in Figure 1, the physical process is not long enough to produce reliable parameters in the clustered ionomers. As reported in the previous publications ${ }^{7-9}$ by Eisenberg and coworkers, the WLF constants based on the "pseudo master curve" are bigger than those obtained from other ionomers with low ion content for which time-temperature superposition is applicable. Naturally, the nature of the secondary process extracted by the "pseudo physical parameters" was somewhat unclear. The activation energy reported previously $^{8}$ for the secondary mechanism, of order of $40 \mathrm{kcal} \mathrm{mol}^{-1}$, also seems to be too high for any conceivable secondary process.

In Figure 2, the same stress relaxation data shown in Figure 1 are normalized by the exponential decay term which is the law for Maxwellian decay of stress ${ }^{1}$ : 


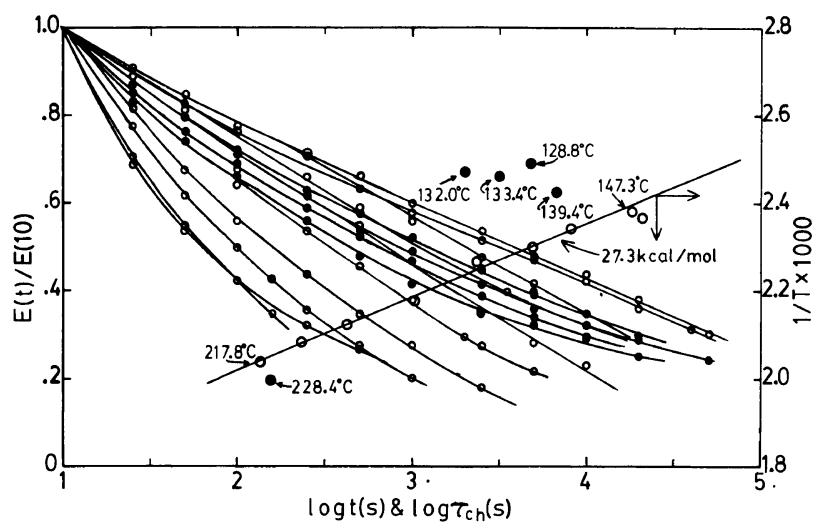

Figure 2. Chemical stress relaxation curves and chemical relaxation times for PS-NaMA $(7.7 \mathrm{~mol} \%$ of NaMA). 10 s modulus as reference. The activation energy is also shown. Some temperatures are omitted for the sake of clarity.

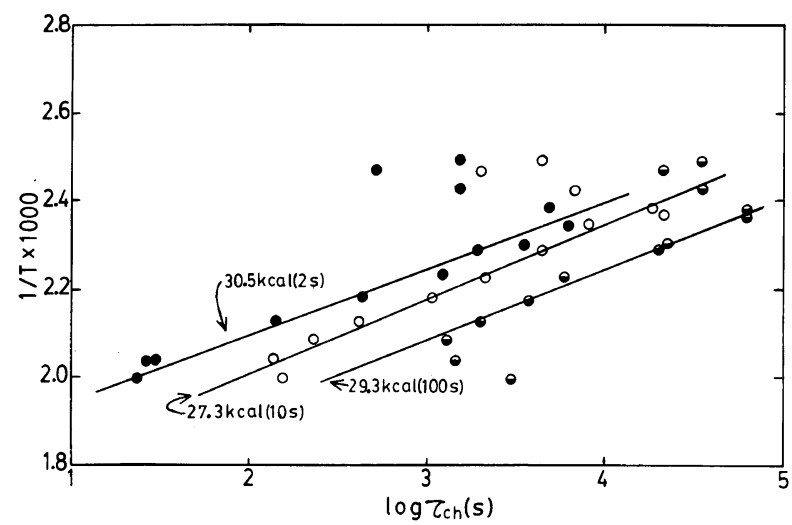

Figure 3. Three different sets of chemical relaxation times, obtained with three different references for PS-NaMA $(7.7 \mathrm{~mol} \%$ of NaMA), are plotted against $1 / T$. For the sake of clarity, chemical relaxation curves are not plotted.

$$
f(t) / f(0) \text { or } E(t) / E(0)=\exp (-t / \tau)
$$

where $\tau$ is a chemical relaxation time, $f$ and $E$ are force and modulus, respectively. $\tau$ may be measured as the time at which $f(t) / f(0)$ or $E(t) / E(0)$ reaches $1 / e(=0.368)$. A series of chemical relaxation times obtained by using $10 \mathrm{~s}$. modulus as reference are plotted against $1 / T$ in Figure 2. It is apparent that chemical relaxation times obtained from the data above around $150^{\circ} \mathrm{C}$ fit the Arrhenius relationship and those from below $150^{\circ} \mathrm{C}$ do not. In order to make this "chemical approach" more general, the same relaxation data are also nor- malized by using both $2 \mathrm{~s}$ and $100 \mathrm{~s}$ moduli as references, respectively, as shown in Figure 3. Neither individual $E(t) / E(2)$ nor $E(t) / E(100)$ is plotted for the sake of clarity in Figure 3. There are some deviations at high temperatures in the case of $E(t) / E(100)$ plot. The "chemical approach," however, seems to be well applicable at temperatures between 150 and $220^{\circ} \mathrm{C}$. The deviations at high temperatures will be discussed in the following publications. ${ }^{16}$

As shown by examples in both Figures 2 and 3 , the contribution from the physical component, most likely from the pure physical 


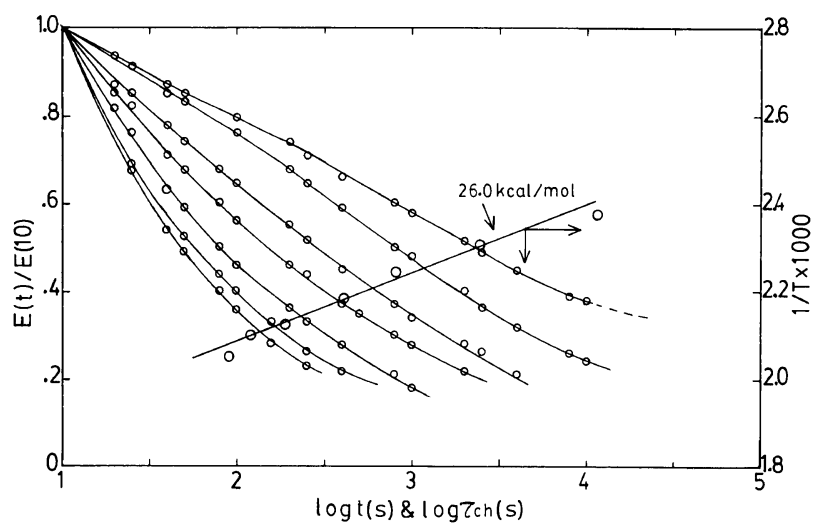

Figure 4. Chemical stress relaxation curves and chemical relaxation times for PS-NaMA $(7.9 \mathrm{~mol} \%)$. Some temperatures are omitted.

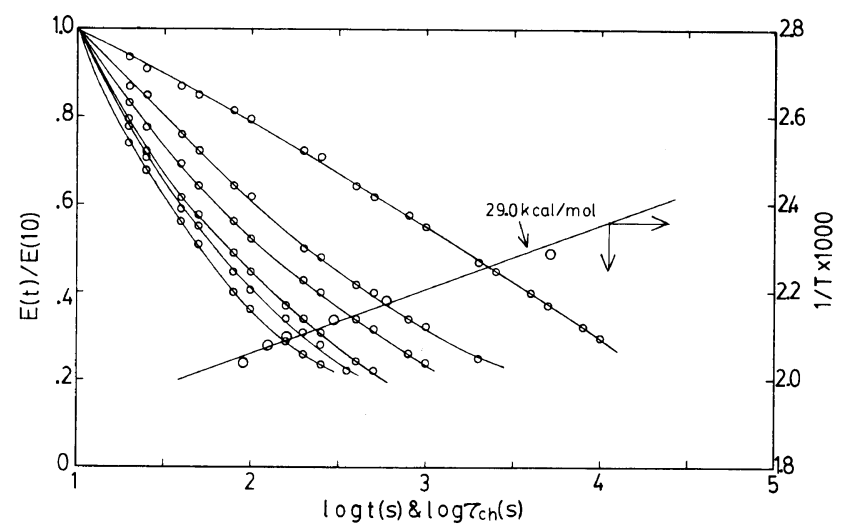

Figure 5. Chemical stress relaxation curves and chemical relaxation times for PS-NaMA $(9.7 \mathrm{~mol} \%)$. Some temperatures are omitted.

one, still exists below $150^{\circ} \mathrm{C}$ or, at least, the physical process becomes less significant above $150^{\circ} \mathrm{C}$. The "chemical approach" is not applicable below $150^{\circ} \mathrm{C}$ in PS-NaMA with $7.7 \mathrm{~mol} \%$ of ions. As a matter of fact, the "chemical approach" is not applicable at all for all PS-NaMA with less than $6 \mathrm{~mol} \%$ of ions. ${ }^{17}$ It is obvious that the physical mechanism is dominant in the ionomers with low ions, especially at low temperatures and in short time regions.

The data from other PS-NaMA with 7.9 and $9.7 \mathrm{~mol} \%$ of ions are normalized the same way and shown in Figures 4 and 5 , respectively. No deviation at low temperatures is observed any longer with these ion- omers. It should be pointed out, however, that the experimental data used here were all originally obtained at temperatures above $150^{\circ} \mathrm{C}$.

The applicability of the "chemical approach" to the clustered ionomers is further demonstrated with PEA-NaA with 16.3. $\mathrm{mol} \%$ of ions. The results from this ionomer are shown in Figure 6. Like the styrene ionomers, the secondary mechanism is also dominant in this acrylate ionomer. In contrast with the case of the styrene ionomers, however, a possible transition temperature of the ionic structure appears at around 50 ${ }^{\circ} \mathrm{C}$, as can be seen in Figure 6. With this figure alone, one may wonder if this is a simple 


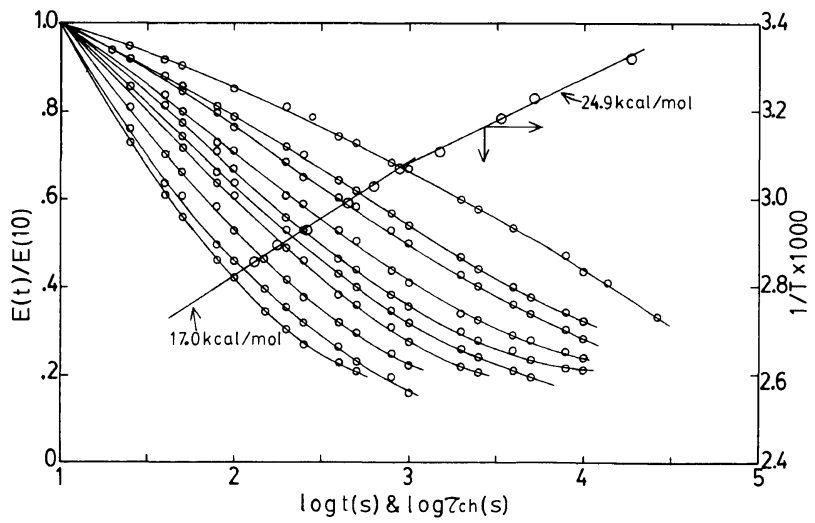

Figure 6. Chemical stress relaxation curves and chemical relaxation times for PEA-NaA $(16.3 \mathrm{~mol} \%$ of $\mathrm{NaA}$ ). " $T_{\mathrm{c}}$ " is observed at around $50^{\circ} \mathrm{C}$.

experimental error or not, but the similar transition points are also observed in other ethyl acrylate ionomers of which results will be published separately. ${ }^{16}$ Although Eisenberg ${ }^{15}$ had long predicted the existence of the decomposition temperature of the ionic structure, $T_{\mathrm{c}}$, it must be the first time that either $T_{\mathrm{c}}$ or some form of transition in the ionic structure has been isolated experimentally. The nature of this transition point will be discussed in the following publication, ${ }^{16}$ too.

The presence of the ionic clusters has been mentioned by several investigators ${ }^{18,19}$ and has also been treated theoretically. ${ }^{15}$ The highly unfavorable thermodynamic situation of ionic salts essentially dissolved in a hydrocarbon medium must lead these ion pairs to segregate themselves and form the ionic clusters. The aggregation of the ionic groups from different polymer chains must also relieve this energetically unfavorable situation. The long-range coulombic interactions between ions undoubtedly assists in setting up the clusters which give rise to a two-phase, reinforced structure. Since the ionic clusters are integrally bonded to the organic phase, these clusters inevitably act as "filler" and "cross-link."

In these ionic clusters, there may be several intermolecular associations involving car- boxyl, carbonyl, and carboxylate groups and cations. The indication for the existence of clusters in the styrene ionomers comes from X-ray evidence. ${ }^{14}$ X-Ray diffraction experiments indicate that above $6 \mathrm{~mol} \%$ cesium salt new scattering centers appear with periodicities of $70-80 \AA$ which can be assigned to a new phase consisting mostly of the ionic clusters.

Metal ions are not permanently associated to any particular carboxyl group. The lifetime of the ionic association must be finite. At higher temperatures and longer times a redistribution of cations can be obtained. Furthermore, under heat and shear, the ionic clusters can dissociate, the ion pairs can diffuse and new ionic clusters may be formed. This would be expected to lead to decay of stress if the sample is deformed. In the ionomers examined here, their relaxation mechanism is approximately Maxwellian, as shown in Figures $2-6$, although it is not very nearly.

The activation energies for the secondary mechanism obtained by the "chemical approach," as also shown in Figures 2-6, are all slightly higher than $25 \mathrm{kcal} \mathrm{mol}^{-1}$. This kind of activation energy is in good agreement with the bond energy of the order of $30 \mathrm{kcal}$ which was calculated by Potts and Aschcraft $^{20}$ for a sodium carboxylate quadruple ion. The basic structure of "quadru- 
ple ions" has been proposed by Erdi and Morawet $\mathrm{z}^{21}$ in the case of styrene-acrylic acid copolymer salts in media of low dielectric constant.

Although it is speculative, it could be assumed that the clustered ionomer behaves essentially like a filled polymer and the ionic clusters act only as inert filler while the physical process is dominant. It is reasonable to assume the contribution from the physical mechanism becomes less and less as temperature increases and finally gets insignificant around $150^{\circ} \mathrm{C}$ in the case of the styrene ionomer with $7.7 \mathrm{~mol} \%$ ions. If the short time regions of the individual curves collected from below $150^{\circ} \mathrm{C}$ were only used to construct a master curve, time-temperature superposition should be applicable because the relaxation mechanism in the short time region is of physical nature. Previously, ${ }^{9,12}$ the short time regions of the individual curves collected from the entire temperature ranges were used to construct the "pseudo master curve" for both ionomer groups. It should be pointed out that, based on the time-temperature superposition principle itself, no secondary process should be observed for the time scale of the experiments at low temperatures if any measurable, pure physical process still existed at high temperatures, $80-100^{\circ} \mathrm{C}$ above its $T_{\mathrm{g}}$ in the case of the styrene ionomers. Actually, Salazar and Curro $^{6}$ determined the WLF constants and other physical parameters at low temperatures with utilizing the difference in relaxation mechanism between high and low temperatures.

As can be seen in Figures 2, 4, and 5, the ionic structures in the styrene ionomers do not completely break down upon heating up to $210-220^{\circ} \mathrm{C}$. Eisenberg and Navratil ${ }^{8,9}$ previously claimed that the ionic clusters broke down around $180^{\circ} \mathrm{C}$ in one of PS-NaMA samples studied here. The ionic structure, however, apparently still exists above $200^{\circ} \mathrm{C}$ for all PS-NaMA samples. Above $220^{\circ} \mathrm{C}$, the ionic structure might break down or change its form, as can be seen in Figure 3. This possibility, however, is highly speculative and remains to be confirmed in further experimentation.

Finally, it should be mentioned for the sake of completeness that some data were omitted when others were normalized because the values of $E(t) / E(0)$ from the omitted ones would never reach $1 / e$. It is, however, certain that results discussed here would never be changed with or without them.

\section{CONCLUSION}

It has been demonstrated, with using a few examples, that the method which was used for the study of the chemical stress relaxation mechanism in covalently bonded polymer networks is a effective way to isolate the secondary mechanism in the clustered ionomers, especially at high temperatures. As shown previously, the inapplicability of time-temperature superposition is most likely due to the absence of the pure physical mechanism or the presence of solid secondary mechanism at high temperatures. The activation energies obtained for the secondary mechanism by the "chemical approach" are all slightly higher than $25 \mathrm{kcal} \mathrm{mol}^{-1}$ which seems to be reasonable for the yielding process of the ionic structures. While the ionic structure of three PS-NaMA samples remains unchanged upon heating up to $220^{\circ} \mathrm{C}$, the ionic structure of PEA-NaA seems to change their form upon heating.

Acknowledgement. The author is indebted to Drs. A. Eisenberg, M. King, and M. Navratil for their comments on this work. The author also wishes to thank Mrs. R. Allen for her critical reading of this paper.

\section{REFERENCES}

1. A. V. Tobolsky, "Properties and Structure of 
Polymers," John Wily, New York, N.Y., 1960.

2. R. C. Osthoff, A. M. Bueche, and W. I. Grubb, J. Am. Chem. Soc., 76, 4659 (1954).

3. K. Murakami and S. Tamura, J. Polym. Sci., A-1, 9, 423 (1971).

4. S. Tamura, Doctoral Thesis, Tohoku University, Sendai, Japan, 1984

5. W. C. Child, Jr. and J. D. Ferry, J. Colloid Sci., 12, 327 (1957).

6. J. G. Curro and E. A. Salazar, J. Appl. Polym. Sci., 19, 2571 (1975).

7. A. Eisenberg and M. Navratil, J. Polym. Sci., B, 10, 537 (1972).

8. M. Navratil, Ph. D. Thesis, McGill University, Montreal, Canada, 1972.

9. A. Eisenberg and M. Navratil, Macromolecules, 6, 604 (1973).

10. M. Navratil and A. Eisenberg, Macromolecules, 7, 84 (1974).

11. A. Eisenberg, M. King, and M. Navratil,
Macromolecules, 6, 734 (1973).

12. A. Eisenberg, H. Matsuura, and T. Tsutsui, $J$. Polym. Sci., B, 18, 479 (1980).

13. H. Matsuura and A. Eisenberg, J. Polym. Sci., B, 14, 1201 (1976).

14. A. Eisenberg and M. Navratil, Macromolecules, 7, 90 (1974).

15. A. Eisenberg, Macromolecules, 3, 147 (1970).

16. H. Matsuura, to be published.

17. H. Matsuura, unpublished results.

18. L. Holding, Ed., "Ionic Polymers," Halstead Press, New York, N.Y., 1975.

19. A. Eisenberg and M. King, "Ion Containing Polymers," Açademic Press, New York, N.Y., 1977.

20. J. E. Potts, E. G. Hendricks, C. Wu, and A. C. Ashcraft, Paper presented at the American Chemical Society, Middle Atlantic Meeting, February 2, 1968.

21. N. Z. Erdi and H. Morawetz, J. Colloid Sci., 19, 708 (1964). 